\title{
SDN Controlled mmWave Massive MIMO Hybrid Precoding for 5G Heterogeneous Mobile Systems
}

\author{
Na Chen, ${ }^{1}$ Songlin Sun, ${ }^{1}$ Michel Kadoch, ${ }^{2}$ and Bo Rong ${ }^{3}$ \\ ${ }^{1}$ School of Information and Communication Engineering, Beijing University of Posts and Telecommunications, Beijing 100876, China \\ ${ }^{2}$ Department of Electrical Engineering, Ecole de Technologie Superieure, Universite du Quebec, Montreal, QC, Canada H3C 1K3 \\ ${ }^{3}$ Communications Research Centre Canada, Ottawa, ON, Canada K2H 8 S2
}

Correspondence should be addressed to Songlin Sun; slsun@bupt.edu.cn

Received 6 November 2015; Revised 1 February 2016; Accepted 9 February 2016

Academic Editor: Qilian Liang

Copyright (c) $2016 \mathrm{Na}$ Chen et al. This is an open access article distributed under the Creative Commons Attribution License, which permits unrestricted use, distribution, and reproduction in any medium, provided the original work is properly cited.

\begin{abstract}
In 5G mobile network, millimeter wave (mmWave) and heterogeneous networks (Hetnets) are significant techniques to sustain coverage and spectral efficiency. In this paper, we utilize the hybrid precoding to overcome hardware constraints on the analogonly beamforming in mmWave systems. Particularly, we identify the complicated antenna coordination and vast spatial domain information as the outstanding challenges in mmWave Hetnets. In our work, we employ software defined network (SDN) to accomplish radio resource management (RRM) and achieve flexible spacial coordination in mmWave Hetnets. In our proposed scheme, SDN controller is responsible for collecting the user channel state information (CSI) and applying hybrid precoding based on the calculated null-space of victim users. Simulation results show that our design can effectively reduce the interference to victim users and support high quality of service.
\end{abstract}

\section{Introduction}

In the past few years, the fourth-generation $(4 \mathrm{G})$ mobile communication systems have achieved rapidly deployment and operated successfully in many countries around the world. Nevertheless, the widespread use of smart phones and tablet personal computers has brought about an increasing demand for mobile data access. Under such a condition, the fifth-generation (5G) mobile system has attracted much attention as a means of providing enhanced connectivity for more diversified devices with higher mobility. 5G mobile system is expected to dominate future implementations of telecommunication networks and its related technologies have become popular research topics.

The major challenge in the $5 \mathrm{G}$ era is to efficiently support the increasing demand of network capacity while the spectrum resource remains scarce [1]. That is to say, the future network is expected to guarantee quality of service (QoS) while handling the complex context of operations characterized by a tenfold increase in traffic [2]. Moreover, service providers are paying more attention to the operating expense (OPEX) and the influence on global climate change, which makes the energy efficiency an urgent issue [3]. Under this condition, operators promote the Hetnet architecture, where a cellular system consists of a large number of densified low power nodes (LPNs) or small cells $[4,5]$. In Hetnets, LPNs can support high data rate to nearby mobile users and therefore improve the system capacity by frequency reuse. Furthermore, LPNs possess significant reduction in energy consumption as they can transmit signals with lower power [6].

MmWave and massive multiple-input multiple-output (MIMO) techniques are also regarded as promising solutions for future $5 \mathrm{G}$ mobile system, for they can provide tremendous increase in both the spectrum efficiency and the available bandwidth [7]. Massive MIMO tends to scale up the conventional MIMO to increase network capacity and mmWave enables a large antenna array to be arranged in a small size as it has short wavelength associated with high frequencies. On the other hand, by exploiting the precoding technique to concentrate the signal in a specific direction, massive MIMO can provide sufficient antenna gains to compensate for the serious signal attenuation brought by mmWave frequencies. 
In mmWave systems, the precoding schemes are mainly performed in the baseband domain where a fully digital precoder is utilized to modify both the amplitude and phase of transmitted signals. Although the fully digital precoding scheme can achieve optimal performance, it brings enormous hardware complexity and energy consumption as it requires an expensive radio frequency (RF) chain for every antenna. Thus, this becomes a serious problem due to the huge number of antennas at the base station (BS) in mmWave systems. Fortunately, the hybrid precoding technique combining digital precoding and analog precoding has been proposed to significantly reduce the number of RF chains, which is considered as an essential technique for realistic mmWave massive MIMO systems [8, 9].

Nevertheless, the development of Hetnets and massive MIMO induces an increasing network complexity which tends to the virtualization of network functionality $[10$, 11]. For example, massive MIMO leads to the problem of managing huge spatial domain resource and processing vast information in mmWave systems. To tackle such conditions, SDN is introduced owing to its inherent advantage in integrating and processing information $[12,13]$.

In our work, we study how SDN benefits the RRM in $5 \mathrm{G}$ mmWave Hetnets. We proposed a network architecture which is consistent with $5 \mathrm{G}$ concepts and also compatible to current 3GPP LTE Advanced (LTE-A) standard. In 4G network, the main issue for RRM is the time and frequency domain management, such as frequency reuse [14], intercell interference coordination (ICIC) [15], and enhanced intercell interference coordination (eICIC). In 5G, however, the system has to reach the overwhelming densities of devices and network infrastructure nodes, as well as supporting an astonishing numbers of antennas in massive MIMO systems [16]. In mmWave 5G Hetnets, there are macrobase station (macro-BS) and small cells all employing multiple antennas which leads to huge spacial domain information. Thus, it becomes a critical task for mmWave Hetnets to retrieve, store, and utilize the spatial information. In our proposed architecture, SDN takes the responsibility for integrating huge spacial domain information and performing null-space calculation concerning massive MIMO coordination. By doing this, the null-space information is integrated to enable whole network coordination and achieves a better performance than the networks without SDN. This novel architecture implements coordinated RRM and eases the operation pressure of access points significantly.

The rest of this paper is organized as follows. Section 2 presents the 5G SDN architecture supporting RRM. Section 3 introduces the mmWave technology and Section 4 emphasizes the mmWave precoding problem. In Section 5, we propose our two-tier interference free mmWave hybrid precoding scheme, followed by the performance evaluation in Section 6. In the end, Section 7 concludes the paper.

\section{SDN Controlled 5G Heterogeneous Mobile System}

In 5G mobile system, Hetnets, massive MIMO, and SDN are expected to be the key technologies sustaining operation

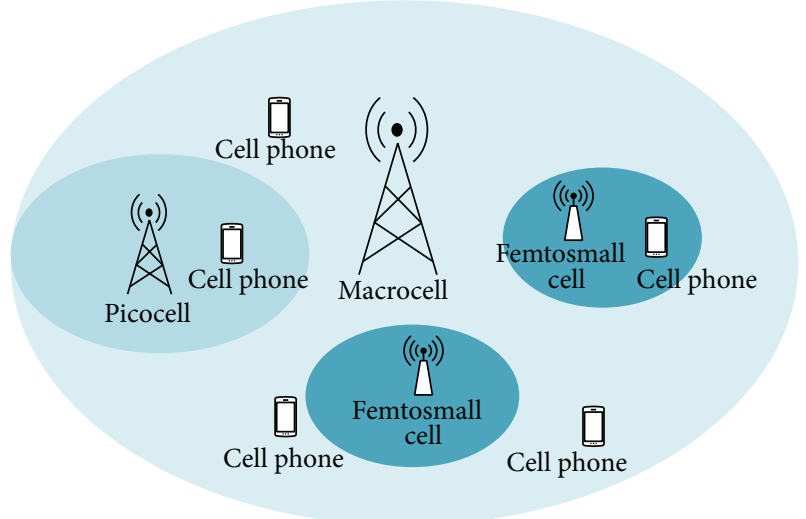

FIGURE 1: A generalized architecture of heterogeneous network.

performance. In this section, we will overview these frontier technologies and introduce our SDN assisted 5G architecture involving mmWave system.

2.1. Hetnet. Hetnet is regarded as the most viable solution to the impending mobile data traffic crunch in the context of LTE-A in the industry. It builds different layers and cell sizes by deploying eNodeBs of different transmission powers, such as macro, micro, pico, and femto as shown in Figure 1. Hetnet becomes an inevitable trend for future development of information networks since it makes full use of the complementary characteristics of different network tiers [17, 18]. Nevertheless, different tiers' networking access technologies and various service requirements make spatial domain management a new problem we have to solve. In $5 \mathrm{G}$ Hetnets, we involve SDN controller to collect and manage spacial information from a variety of access points.

2.2. Massive MIMO. In massive MIMO systems, the antenna arrays consisting of a few hundred elements simultaneously serve many tens of mobile users with the same timefrequency resource [19]. Massive MIMO is an enabler for future broadband (fixed and mobile) networks, which will be energy-efficient, spectrum-efficient, and robust. However, massive MIMO antenna arrays engender huge amounts of spacial domain information in real time, which significantly increases the computational complexity and brings about the massive MIMO coordination problem. The network can operate well only when SDN processes spacial information successfully.

2.3. SDN. The concept of SDN was first introduced in the 1990 s and became popular in the 21st century. The architecture of SDN was formally defined by the open networking forum (ONF). In SDN architecture, network devices consist of three layers, that is, application layer, control layer, and infrastructure layer [20].

Currently, SDN has found its best practice in data centers and has been paid increasing attention by network organizations around the world [21]. SDN is regarded as a promising method to solve current and emerging problems for future $5 \mathrm{G}$ 


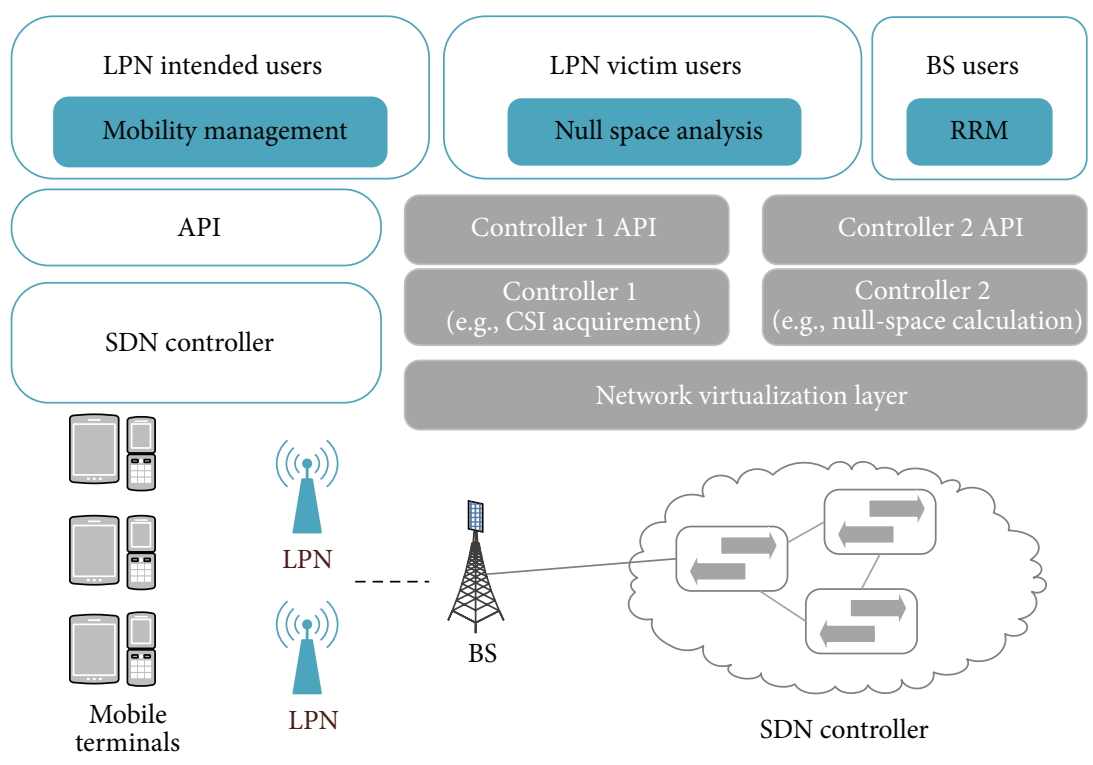

FIGURE 2: 5G SDN architecture for spatial domain management.

Hetnets [22, 23]. Figure 2 illustrates our proposed architecture, where an SDN controller is employed to manage spatial information and conduct massive MIMO coordination for $5 \mathrm{G}$ network. In this architecture, user information is first collected by BS/LPNs and then reported to SDN. SDN controller will perform the information processing such as CSI analysis and null-space calculation. SDN controller then sends the processed results and instructions back to BS/LPNs to perform better coordination concerning the global knowledge of network spatial information. In this architecture, SDN takes over the heavy operation from BS and LPNs to increase the efficiency of $5 \mathrm{G}$ networks.

\section{Millimeter Wave Technology}

3.1. Millimeter Wave Introduction. Millimeter wave is a promising technology for future 5G cellular networks. In core network, the mmWave system is expected to bring highcapacity wireless access networks in the future [24]. Future network equipped with mmWave can achieve super wide bandwidth, as the frequency of mmWave ranges from 26.5 to $300 \mathrm{GHz}$. Moreover, mmWave has a much narrower antenna beam size compared to the microwave; therefore, it can aim at the target more precisely. Practically, mmWave cellular systems can not alone provide high quality service across a range of deployments. Thus, mmWave network is generally heterogeneous due to the inherent limitations of mmWave propagation. As shown in Figure 3, it is quite likely that local area networks and cellular network will blur over time.

3.2. $5 G$ mmWave Hetnet. The current $4 \mathrm{G}$ cellular networks are capable of providing reliable communications and seamless coverage because of the lower frequency band they use. In order to implement smooth and cost-efficient transition from $4 \mathrm{G}$ to $5 \mathrm{G}$, one of the solutions is to deploy $4 \mathrm{G}+$

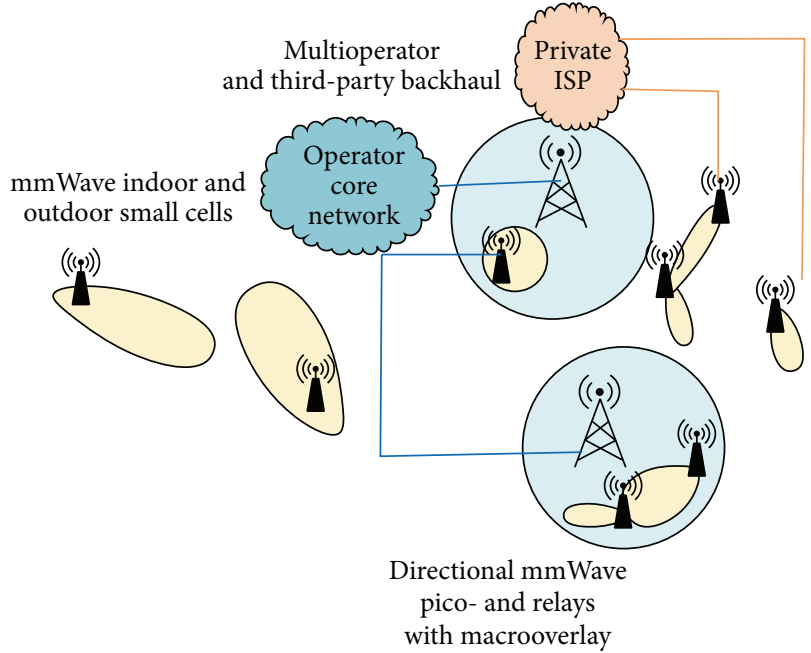

FIGURE 3: Future network implementing mmWave systems.

mmWave system structure in $5 \mathrm{G}$ Hetnets as illustrated in Figure 4. This $5 \mathrm{G}$ Hetnet architecture consists of $4 \mathrm{G}$ base stations, mmWave base stations, and mobile devices. In this architecture of $4 \mathrm{G}+$ mmWave, $4 \mathrm{G}$ network is responsible for transmitting management information and low rate data like text and voice. Meanwhile, the mmWave band takes over the transmission of high-rate multimedia applications $[1,25]$.

\section{Millimeter Wave Precoding}

4.1. mmWave Precoding Problems. With a large number of antennas, linear precoding has low complexity and comparable performance to its counterpart nonlinear precoding. However, MIMO precoding in mmWave systems is generally different than the precoding at lower frequencies. To exploit 


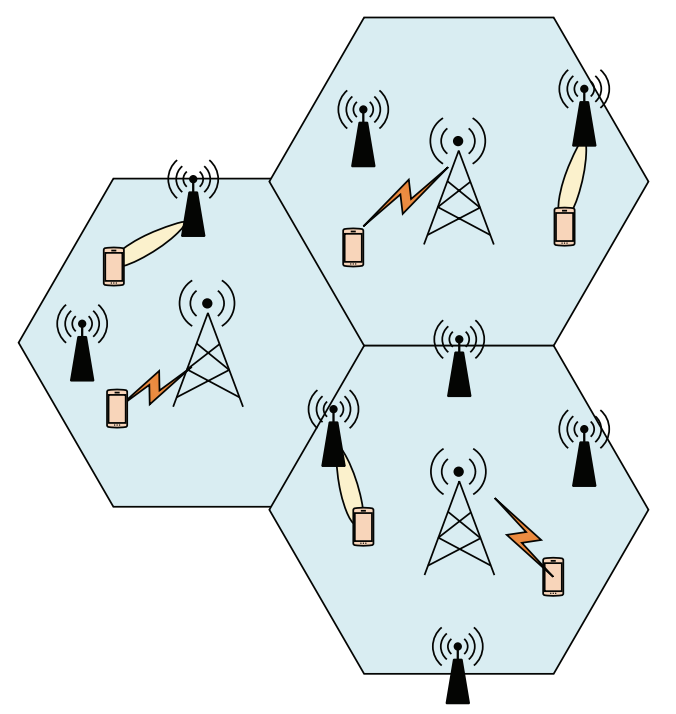

$((\bullet))$

8

$(((\mathrm{p})))$ mmWave base station

Mobile device

FIgURE 4: The 4G + mmWave system structure.

multiple antennas, the conventional precoding schemes tend to modify the amplitudes and phases of the complex symbols at the baseband and then upconvert the processed signal to around the carrier frequency after passing through digitalto-analog (D/A) converters, mixers, and power amplifiers (i.e., RF chains). Thus, each antenna element needs to be supported by a dedicated RF chain. Actually, this is too expensive to be implemented in mmWave systems due to the large number of antennas [1].

4.2. Hybrid Precoding. To solve the above problem, hybrid precoding structure with active antennas is considered, which generally controls the signal phase on each antenna via a network of analog phase shifters. As illustrated in Figure 5, a hybrid precoding structure is divided into analog and digital domains. Firstly, user data streams are precoded by the baseband digital processing. Then, the streams are transferred by the corresponding RF chains and mapped into each antenna element by the analog phase processing (RF processing). In the process, the number of RF chains is lower than that of antennas, which reduces the complexity and power consumption in mmWave system. Notably, baseband digital processing can make both amplitude and phase modifications, but analog phase processing only gets the phase changes with variable phase shifters and combiners [15].

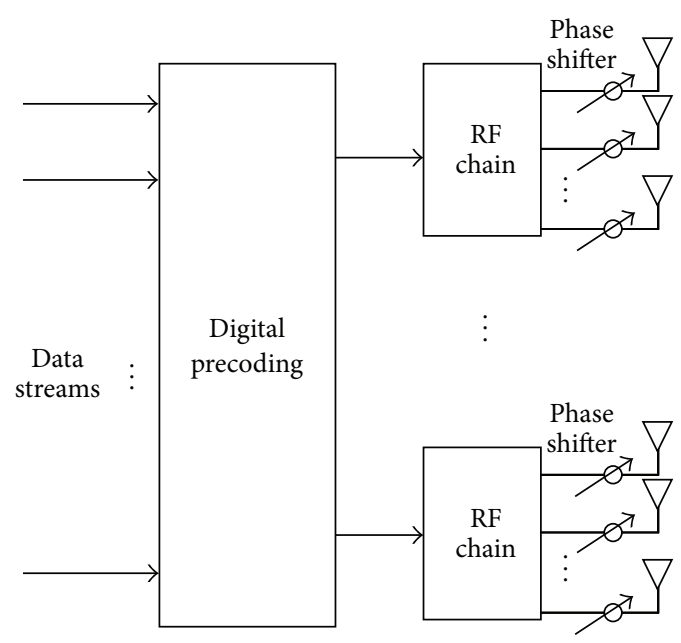

FIGURE 5: Base station hybrid precoding arrangement.

\section{Two-Tier Interference Free mmWave Hybrid Precoding Scheme}

In this section, we propose our scheme to implement effective RRM in mmWave $5 \mathrm{G}$ network. Here, we use null-space based hybrid precoding to overcome the constraints and mitigate intertier interference in mmWave system. We also distinguish our work by involving an SDN controller to handle massive MIMO coordination for the whole $5 \mathrm{G}$ network.

5.1. System Model. Let us assume a downlink mmWave system with $K$ single-antenna users as shown in Figure 8 . The $\mathrm{BS}$ and LPNs are all equipped with massive MIMO containing $N_{\mathrm{BS}}$ and $N$ transmit antennas, respectively. Let $K_{j}$ denote the number of users of the $j$ th LPN. Define $C_{j}$ as the number of users interfered by the $j$ th LPN and served by other nodes. $K_{j}$ and $C_{j}$ should meet the constraint $K_{j}+C_{j} \leq N$. Thus, the stationary channel $\mathbf{H}_{j} \in \mathbb{C}^{\left(K_{j}+C_{j}\right) \times N}$ between a certain LPN $j$ and the users it serves is

$$
\mathbf{H}_{j}=\left[\begin{array}{c}
\mathbf{H}_{j, 1} \\
\vdots \\
\mathbf{H}_{j, K_{j}} \\
\mathbf{H}_{j, K_{j}+1} \\
\vdots \\
\mathbf{H}_{j, K_{j}+C_{j}}
\end{array}\right],
$$

where the $k$ th row vector $\mathbf{H}_{j, k} \in \mathbb{C}^{1 \times N}$ is the channel between the $j$ th LPN and the $k$ th user. Specially, $\mathbf{H}_{0}$ denotes the stationary channel between macro-BS and the users it serves. $\mathbf{H}_{0, k}$ denotes the channel between the macro-BS and the $k$ th user. 


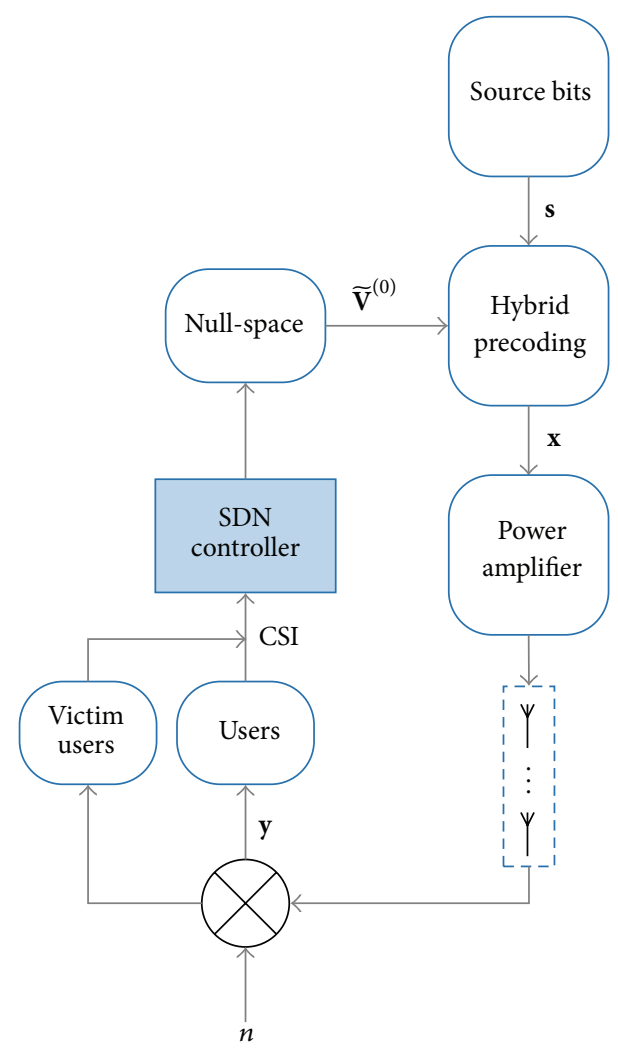

FIGURE 6: Block diagram of our proposed scheme.

Based on the proposed SDN architecture mentioned above, Figure 6 illustrates our processing diagram emphasizing the acquisition of CSI for LPNs and the generation of nullspace based hybrid precoding matrix. And we will expound the details in the following.

5.2. CSI Acquisition Method. In practice, LPNs is only able to collect the local CSI through the backward channel of their own served users. Thus, they can not access to the CSI of the external victim users interfered by them. Fortunately, the hub-spoke structure of the SDN network enables the CSI of all mmWave channels to be collected and disseminated through the backhaul link [26].

In our proposed CSI acquisition method as illustrated in Figure 7, BS collects and reports the CSI information to the SDN controller. More exactly, BS will send SDN the channel matrix on victim users of each LPN, which means sending SDN every $\left[\mathbf{H}_{j, K_{j}+1}^{H}, \ldots, \mathbf{H}_{j, K_{j}+C_{j}}^{H}\right]^{H}$ corresponding to the $j$ th LPN. Then, the SDN controller will perform the blockdiagonal (BD) algorithm and generate null-space vector $\widetilde{\mathbf{V}}_{j, v}^{(0)}$ for LPN $j$. After that, the SDN controller computes on the null-space vector $\widetilde{\mathbf{V}}_{j, v}^{(0)}$ to gain precoding matrix by the hybrid precoding algorithm proposed in the next subsection. Finally, SDN controller sends the precoding matrix to each LPN through downlink information. In this way, the SDN controller takes over all the matrix decomposition computation and precoding. This framework is more helpful when the LPNs are too simple to handle the computational work.

Evidently, when the precoding vectors span a subspace of the null-space of the channel vectors from victim users, the interferences from the intended users can not affect the victim users. According to this, the hybrid precoding matrix can be constructed by processing the mmWave channel matrix.

5.3. Hybrid Precoding Based on Null-Space. Hybrid precoding is divided among baseband and RF processing, denoted by $\mathbf{D} \in \mathbb{C}^{K \times K}$ and $\mathbf{A} \in \mathbb{C}^{N \times K}$ as illustrated in Figure 8 .

Each entry of $\mathbf{A}$ is normalised to satisfy $\left|\mathbf{A}_{a, b}\right|=1 / \sqrt{N}$, where $\left|\mathbf{A}_{a, b}\right|$ denotes the magnitude of the $(a, b)$ th element of $\mathbf{A}$. To clarify, denote $\mathbf{A}_{a, b}$ as the $(a, b)$ th element of $\mathbf{A}$ and we perform the RF precoding according to

$$
\mathbf{A}_{a, b}=\frac{1}{\sqrt{N}} e^{b \varphi_{a, b}},
$$

where $\varphi_{a, b}$ is the phase of the $(a, b)$ th element of the conjugate transpose of the stationary channel, that is, $\mathbf{H}^{H}$. At the baseband, we observe an equivalent channel $\mathbf{H}_{\mathrm{eq}}=\mathbf{H A}$ of a low dimension $K \times K$, where $\mathbf{H}$ is the stationary channel. We use a ZF precoding as the digital precoding algorithm; thus, baseband precoding is performed as

$$
\mathbf{D}=\mathbf{H}_{\mathrm{eq}}^{H}\left(\mathbf{H}_{\mathrm{eq}} \mathbf{H}_{\mathrm{eq}}^{H}\right)^{-1} \boldsymbol{\Lambda},
$$

where $\boldsymbol{\Lambda}$ is a diagonal matrix introducing for column power normalization.

The received signal of the $k$ th user can be written as

$$
y_{j, k}=\mathbf{H}_{j, k} \mathbf{A}_{j} \mathbf{D}_{j} \mathbf{s}_{j, k}+\mathbf{H}_{0, k} \mathbf{A}_{0} \mathbf{D}_{0} s_{0}+n_{j, k}
$$

where $\mathbf{s}_{j} \in \mathbb{C}^{K_{j} \times 1}$ is the transmitted signal vector for a total of $K_{j}$ users. And $\mathbf{s}_{0}$ is the transmitted signal vector at BS. $n_{j, k}$ is the additive white Gaussian noise of zero mean and variance $\sigma^{2}$.

For the $j$ th LPN, the complementary space concerning the victim users is given as

$$
\widetilde{\mathbf{H}}_{j, v}=\left[\mathbf{H}_{j, K_{j}+1}^{H} \cdots \mathbf{H}_{j, K_{j}+C_{j}}^{H}\right]^{H},
$$

where $\widetilde{\mathbf{H}}_{j, v}$ contains the channel matrix of all the victim users interfered by the $j$ th LPN. To avoid the interference to the victim users, the hybrid precoding matrix should satisfy the following condition:

$$
\widetilde{\mathbf{H}}_{j, v} \mathbf{A}_{j} \mathbf{D}_{j}=\mathbf{0}^{C_{j} \times K_{j}} .
$$

By performing SVD, $\widetilde{\mathbf{H}}_{j, v}$ can be further written as

$$
\begin{aligned}
\widetilde{\mathbf{H}}_{j, v} & =\widetilde{\mathbf{U}}_{j, v} \widetilde{\boldsymbol{\Lambda}}_{j, v} \widetilde{\mathbf{v}}_{j, v}^{H} \\
& =\widetilde{\mathbf{U}}_{j, v}\left[\sum_{j, v} \mathbf{0}\right]_{C_{j} \times N}\left[\widetilde{\mathbf{v}}_{j, v, 1} \widetilde{\mathbf{v}}_{j, v, 2} \cdots \widetilde{\mathbf{v}}_{j, v, N}\right]^{H} .
\end{aligned}
$$




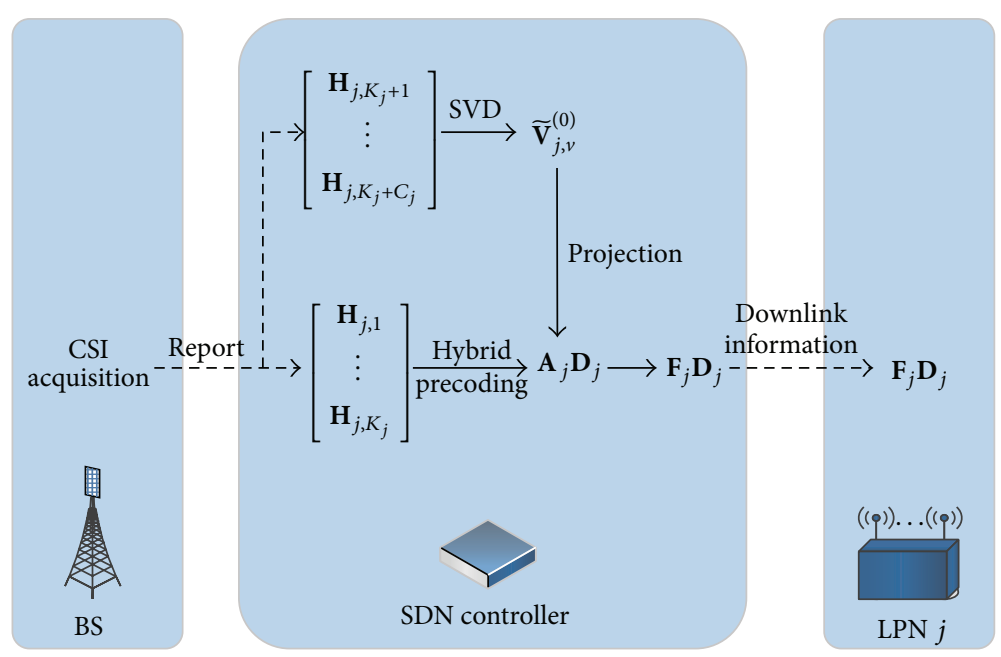

FIGURE 7: Proposed CSI acquirement methods.

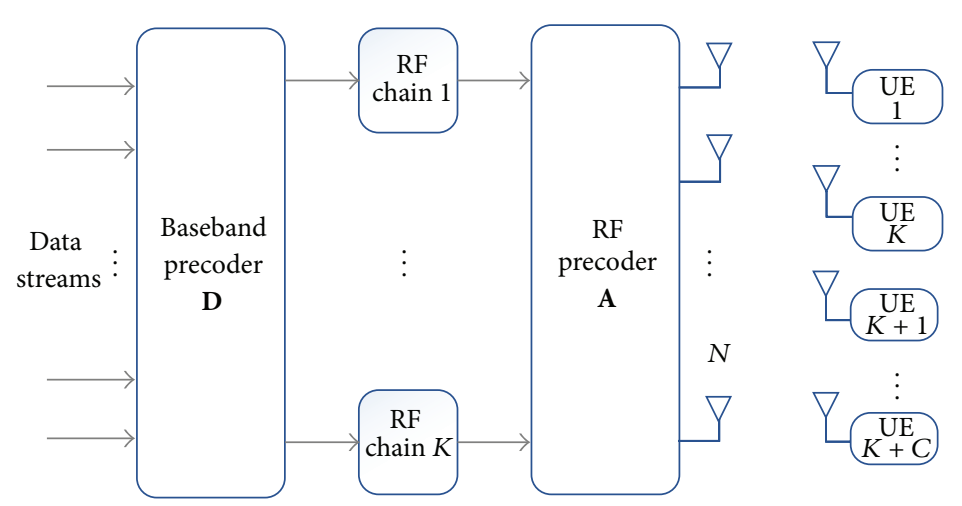

FIGURE 8: Hybrid mmWave precoding structure.

Define the null-space vector $\widetilde{\mathbf{V}}_{j, v}^{(0)}=\left[\widetilde{\mathbf{v}}_{j, v, C_{j}+1} \cdots \widetilde{\mathbf{v}}_{j, v, N}\right]$. Since the column vectors belonging to $\widetilde{\mathbf{V}}_{j, v}^{(0)}$ locate in the nullspace of all victim users, we will have $\widetilde{\mathbf{H}}_{j, v} \widetilde{\mathbf{V}}_{j, v}^{(0)}=0$.

For the $j$ th LPN, define the projection matrix $\mathbf{M}_{j}$ based on the null-space of victim users by

$$
\mathbf{M}_{j}=\tilde{\mathbf{V}}_{j, v}^{(0)}\left(\tilde{\mathbf{V}}_{j, v}^{(0)}\right)^{H}
$$

The new analog precoding matrix after projection can be calculated as

$$
\mathbf{F}_{j}=\mathbf{M}_{j} \mathbf{A}_{j} .
$$

Define $P_{j}$ as the transmit power at the $j$ th LPN satisfying $E\left[\mathbf{s}_{j} \mathbf{s}_{j}^{H}\right]=\left(P_{j} / K_{j}\right) \mathbf{I}_{K_{j}}$, where $\mathbf{s}_{j}$ denotes the signal vector for $K_{j}$ users in total. We further normalize $\mathbf{F}_{j}$ to satisfy $\left\|\mathbf{F}_{j} \mathbf{D}_{j}\right\|_{F}^{2}=$ $K_{j}$ for the total transmit power constraint.
Thus, the SINR of the $k$ th user served by LPN $j$ is given as

$$
\operatorname{SINR}_{j, k}=\frac{\left(P_{j} / K_{j}\right)\left|\mathbf{H}_{j, k} \mathbf{F}_{j} \mathbf{d}_{j, k}\right|^{2}}{\sigma_{j, k}^{2}+\sum_{r=1, r \neq k}^{K_{j}}\left(P_{j} / K_{j}\right)\left|\mathbf{H}_{j, k} \mathbf{F}_{j} \mathbf{d}_{j, r}\right|^{2}}
$$

where $\mathbf{d}_{j, r}$ denotes the $r$ th column of $\mathbf{D}_{j}$.

To support each user's QoS requirement, the SINR should meet

$$
B \log \left(1+\operatorname{SINR}_{j, k}\right) \geq R_{j, k}
$$

for $j=1,2, \ldots, J$ and $k=1,2, \ldots, K_{j}$, where $R_{j, k}$ is the data rate demanded by user $k$ and $B$ is the total bandwidth. Then, the minimum $\mathbf{F}_{j, k}$ can be solved by the following linear equation system:

$$
B \log \left(1+\operatorname{SINR}_{j, k}\right)=R_{j, k} .
$$




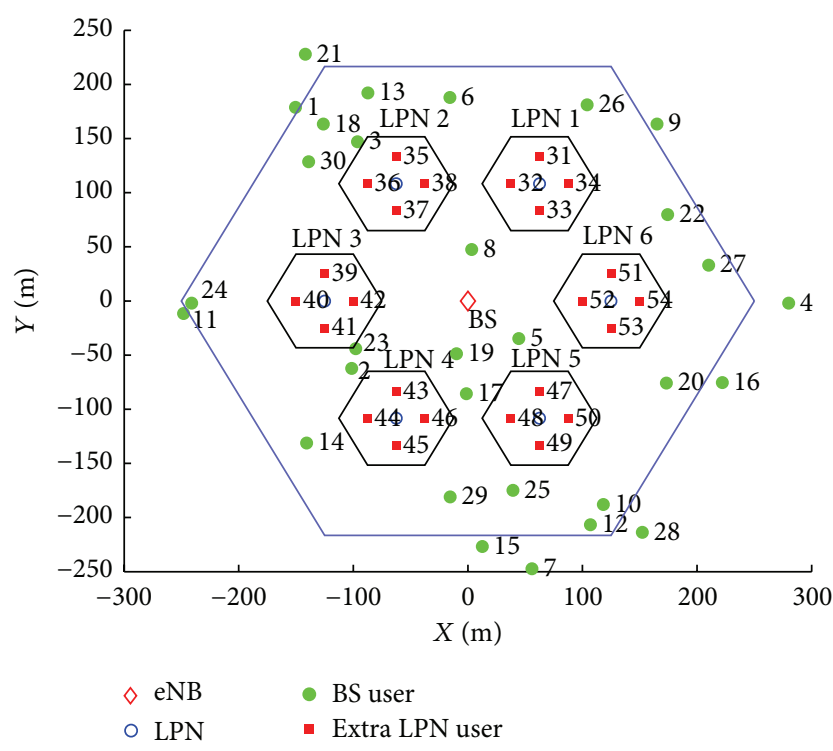

(a) The topology of the macrocell

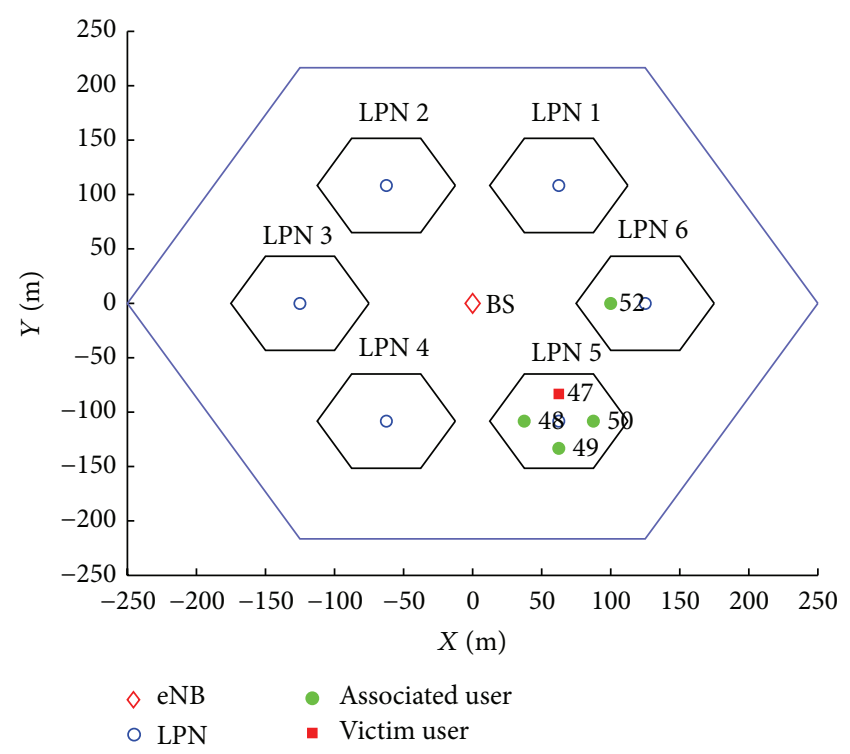

(b) The topology of the LPN 1

FIGURE 9: The overall topology.

5.4. Improvements of Our Proposed Algorithm. Comparing with the conventional linear precoding schemes, the proposed null-space based hybrid precoding algorithm has the following improvements.

5.4.1. SDN Aided CSI Acquisition. In conventional Hetnets communication system, the served user equipment reports its CSI to the connected access point, but not to the other nodes interfering with it. In this case, LPNs can not detect the victim users' CSI. However, by performing our proposed algorithm, the SDN controller can get enough information to form the precoding vector that avoids the interference with the victim users. Furthermore, the computational burden on LPN can be relaxed by letting SDN perform the null-space construction [27].

5.4.2. Reduced Complexity. Conventional linear precoding schemes typically require $N$ RF chains which means tremendous hardware complexity. By involving hybrid precoding, the number of RF chains in mmWave system is reduced from antennas scale to users scale. Therefore, the proposed hybrid precoding scheme owns a much lower complexity than conventional linear precoding schemes. In practice, our proposed scheme can significantly reduce the complexity and power consumption in $5 \mathrm{G}$ mmWave Hetnets.

5.4.3. Improved QoS. By performing hybrid precoding based on null-space of the victim users, the interference with the victim users can be totally avoided, for the channel matrix of victim users and the generated precoding vectors are orthogonal to each other. Meanwhile, the SINR calculation is adjusted to further conform to the practice. Thus, it can effectively mitigate the interference to achieve improved QoS for users with satisfying power consumption, cooperating with hybrid precoding algorithm.

\section{Simulation Results}

To evaluate the performance of our proposed hybrid precoding scheme, this section presents the numerical results based on simulations in 5G Hetnets scenario. We apply the network topology that combines mmWave system and small cells and implement our proposed algorithm to achieve target QoS with low complexity. We deploy the hybrid precoding to reduce hardware complexity in mmWave systems and take the property of null-space to mitigate the interference with the victim users.

Figure 9 illustrates the simulation scenario where the intended users are marked in red and victim users in green. Meanwhile, we analyze the system performance over different simulation parameters and channel realizations. Table 1 displays the main simulation parameters which characterize the macrocell and the LPNs.

The channels model is similar to that for heterogeneous deployments suggested by the 3GPP LTE standard, but the small-scale fading is assumed to be Rayleigh according to recent works on massive MIMO. The correlation matrix between the BS and each user is modeled according to the physical channel model, where the main characteristics are antenna correlation, and reduces rank channels.

In the following, we will present the simulation results in the the scenario as described in Figure 9, in which each user has the QoS demand of $20 \mathrm{Mbps}$. In the proposed precoding scheme, SDN performs the null-space construction and transmits the corresponding part of the precoding vector to the LPNs as described in Section 4. For comparison purposes, we also simulated two conventional linear precoding 
TABLE 1: Simulation parameters.

\begin{tabular}{lc}
\hline Parameters & Values \\
\hline Number of the macrocell users & $N_{\mathrm{BS}} \in\{20,28,36, \ldots, 60\}, 42$ \\
Antennas of the macrocell & $N_{\mathrm{BS}} \in\{52,56,60, \ldots, 112\}, 50$ \\
Antennas of the LPNs & $N_{\mathrm{LPN}} \in\{4,5,6,8\}$ \\
Macrocell radius & $500 \mathrm{~m}$ \\
LPNs radius & $50 \mathrm{~m}$ \\
Total bandwidth & $1 \mathrm{Ghz}$ \\
Number of per LPN users & 4 \\
\hline
\end{tabular}

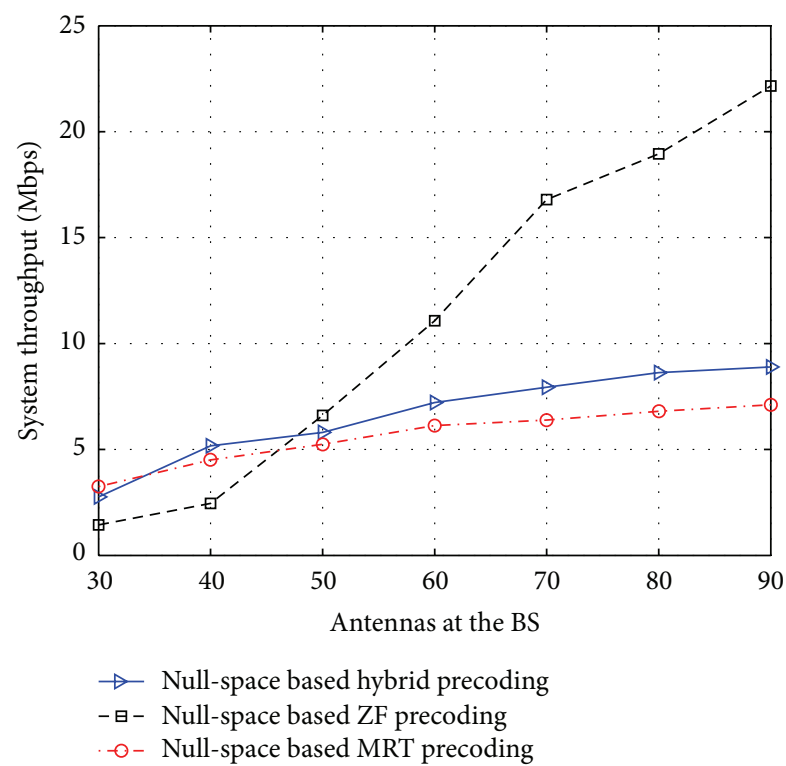

FIGURE 10: System throughput of the different number of antennas.

schemes, max ratio transmission (MRT) and ZF, which are null-space based as well. Theoretically, ZF precoding will have the optimal performance. The simulation results contain both the conventional linear precoding schemes and the proposed hybrid precoding scheme.

6.1. System Throughput by Different Number of Antennas. Figure 10 shows the system throughput effected by the number of massive MIMO antennas at access points. We compare the proposed hybrid precoding scheme to the linear ZF and MRT precoding schemes which all combine the null-space method to mitigate inner-tier interference. Massive MIMO showed a performance improvement in system throughput and the increased antennas can eliminate the interference with the victim users. We can see that the null-space based hybrid precoding scheme gains considerable throughput comparing to the optimal ZF precoding and overwhelms the MRT precoding when antennas increase. This means that our proposed precoding scheme can offer a complexity reduction at a comparable performance [28].

6.2. Spectral Efficiency by Different SNR. Figure 11 compares the spectral efficiency of the conventional linear precoding

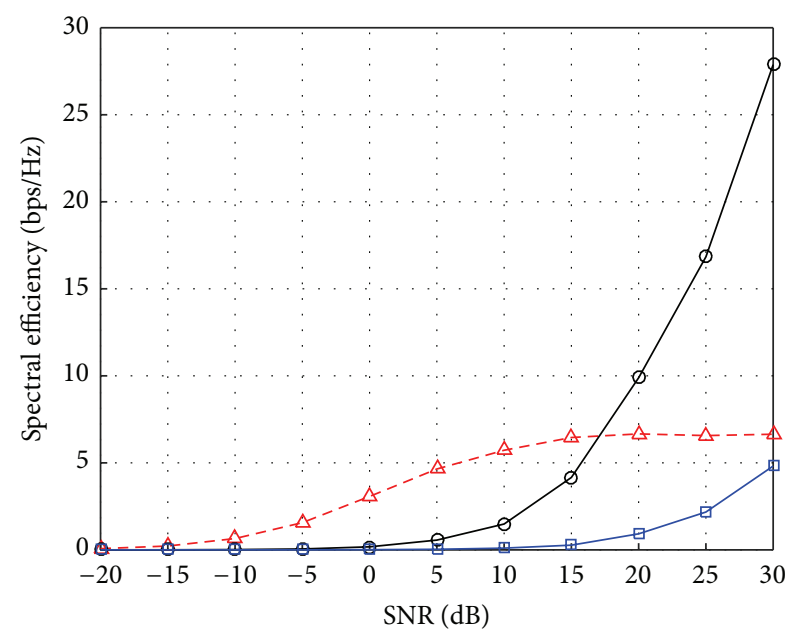

- - Null-space based hybrid precoding

- Null-space based ZF precoding

$\square$ Null-space based MRT precoding

FIGURE 11: Spectral efficiency of different SNR of users.

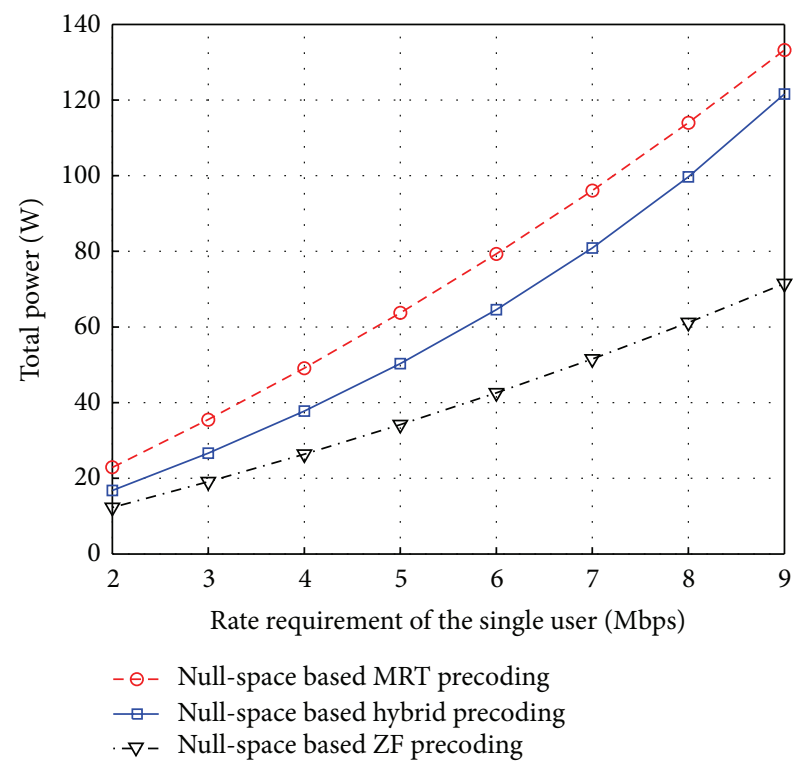

FIgURE 12: Impact of the different QoS requirements.

schemes and the proposed hybrid one under different SNR conditions. We can see that the hybrid precoding scheme has a higher spectral efficiency than the MRT precoding. It means that this hybrid precoding scheme is suitable for realistic mmWave system since it is physically practical and gains comparable spectral efficiency.

6.3. Utility of Different Algorithms Impacted by Different QoS Requirements. Figure 12 compares the different algorithms under different QoS requirements. As we can see, the nullspace based hybrid precoding scheme is still comparable to the ZF precoding and superior to the MRT precoding in the power consumption aspect. Figure 12 also shows 


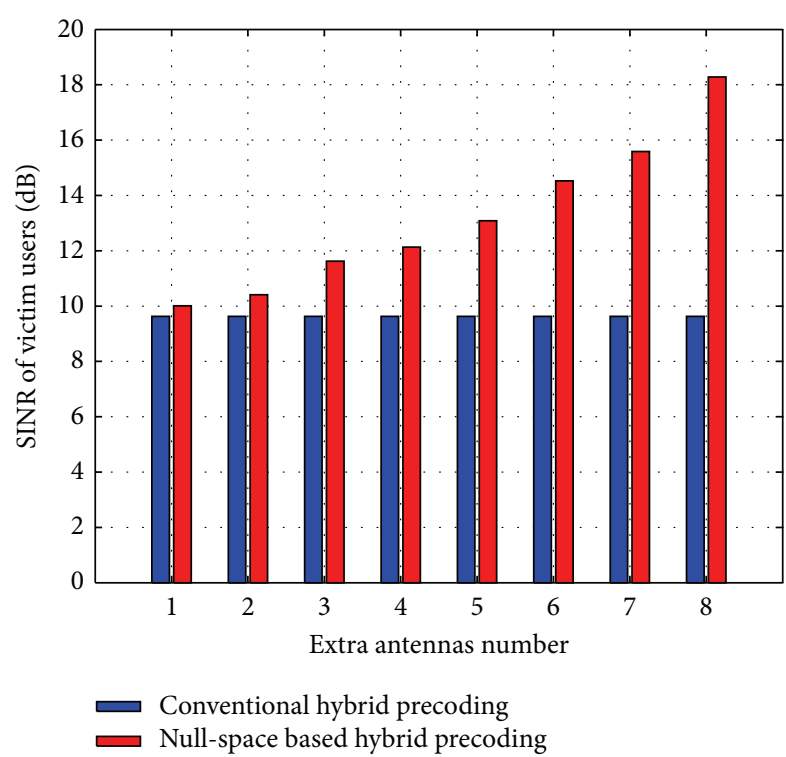

Figure 13: Effect of the extra antennas.

the relationship between the user QoS and the corresponding power consumption. By using the null-space based precoding schemes, the interference of the other BS/LPNs with the victim users can be totally eliminated, which consumes less power than the non-null-space based ones [29].

\subsection{SINR Effect by Different Number of Extra Antennas.} Figure 13 illustrates the SINR distribution of the conventional hybrid precoding scheme [29] and the proposed null-space based one. The hybrid precoding scheme, named phasedZF (PZF), essentially applies phase-only control at the RF domain and then performs a low-dimensional baseband zero-forcing $(\mathrm{ZF})$ precoding based on the effective channel seen from baseband. As we can see, the proposed scheme has a better SINR, which indicates an improved system performance. The difference between these two schemes' performances is due to the fact that the victim users encounter strong interference from other nodes in the conventional scheme, while the proposed hybrid precoding scheme utilises the extra antennas to indicate victim users and then project its users to the corresponding null-space. Hence, it eliminates the strong interference to the victim users and the SINR of the victim users will increase. In Figure 13, the access points are not equipped with sufficient antennas and the interference can only be partially removed. The extra antennas can indicate more victim users and therefore the new system can eliminate more interference. When there are sufficient antennas, it can totally eliminate the interference from other BS/LPNs. This means that having more extra antennas contributes to achieving higher SINR significantly.

In general, the proposed mmWave precoding scheme can drastically alleviate the interference and maintain a desirable system performance for next-generation Hetnets. The projection to the null-space protects the victim users and the combined hybrid precoding algorithms reduce hardware complexity in mmWave systems. Comparing to the conventional design, the new scheme is practical and has a satisfying system throughput with low complexity by taking advantage of the SDN aided CSI acquisition.

\section{Conclusion}

In this paper, we focus on the spatial domain resource management and interference mitigation in mmWave Hetnets. We have explored a centralized 5G architecture using SDN technology to provide coordinated RRM for the whole network. Moreover, we have proposed a lightweight CSI acquisition method and a null-space based hybrid precoding scheme. Our work can significantly oppress the impact on neighboring victim users in LPN-covered small cells. Simulation results show that our proposed design can support sufficient network capacity and improve SINR of the small cell users. This design also has an advantage in practice as it overcomes the physical constraints in mmWave system.

\section{Conflict of Interests}

The authors declare that there is no conflict of interests regarding the publication of this paper.

\section{Acknowledgment}

This work is supported in part by National Natural Science Foundation of China (NSFC) under Grant no. 61471066 and the scholarship from China Scholarship Council (CSC) under Grant CSC no. 201506470023.

\section{References}

[1] R. Q. Hu and Y. Qian, "An energy efficient and spectrum efficient wireless heterogeneous network framework for $5 \mathrm{G}$ systems," IEEE Communications Magazine, vol. 52, no. 5, pp. 94101, 2014.

[2] M. Palkovic, P. Raghavan, M. Li, A. Dejonghe, L. Van der Perre, and F. Catthoor, "Future software-defined radio platforms and mapping flows," IEEE Signal Processing Magazine, vol. 27, no. 2, pp. 22-33, 2010.

[3] I. Chih-Lin, C. Rowell, S. Han, Z. Xu, G. Li, and Z. Pan, "Toward green and soft: a $5 \mathrm{G}$ perspective," IEEE Communications Magazine, vol. 52, no. 2, pp. 66-73, 2014.

[4] R. Q. Hu, Y. Qian, S. Kota, and G. Giambene, "Hetnets-a new paradigm for increasing cellular capacity and coverage," IEEE Wireless Communications, vol. 18, no. 3, pp. 8-9, 2011.

[5] R. Q. Hu and Y. Qian, Heterogeneous Cellular Networks, John Wiley \& Sons, Hoboken, NJ, USA, 2013.

[6] X. Duan and X. Wang, "Authentication handover and privacy protection in 5G hetnets using software-defined networking," IEEE Communications Magazine, vol. 53, no. 4, pp. 28-35, 2015.

[7] E. G. Larsson, O. Edfors, F. Tufvesson, and T. L. Marzetta, "Massive MIMO for next generation wireless systems," IEEE Communications Magazine, vol. 52, no. 2, pp. 186-195, 2014.

[8] A. Alkhateeb, O. El Ayach, G. Leus, and R. W. Heath, "Channel estimation and hybrid precoding for millimeter wave cellular systems," IEEE Journal on Selected Topics in Signal Processing, vol. 8, no. 5, pp. 831-846, 2014. 
[9] A. Alkhateeb, J. Mo, N. González-Prelcic, and R. W. Heath Jr., "MIMO precoding and combining solutions for millimeterwave systems," IEEE Communications Magazine, vol. 52, no. 12, pp. 122-131, 2014.

[10] V. Jungnickel, K. Manolakis, W. Zirwas et al., "The role of small cells, coordinated multipoint, and massive MIMO in 5G," IEEE Communications Magazine, vol. 52, no. 5, pp. 44-51, 2014.

[11] B. Panzner, W. Zirwas, S. Dierks et al., "Deployment and implementation strategies for massive MIMO in 5G," in Proceedings of the IEEE Globecom Workshops (GC Wkshps '14), pp. 346-351, Austin, Tex, USA, December 2014.

[12] C.-F. Lai, R.-H. Hwang, H.-C. Chao, M. M. Hassan, and A. Alamri, "A buffer-aware HTTP live streaming approach for SDN-enabled 5G wireless networks," IEEE Network, vol. 29, no. 1, pp. 49-55, 2015.

[13] P. Ameigeiras, J. J. Ramos-Munoz, L. Schumacher, J. PradosGarzon, J. Navarro-Ortiz, and J. M. Lopez-Soler, "Link-level access cloud architecture design based on SDN for $5 \mathrm{G}$ networks," IEEE Network, vol. 29, no. 2, pp. 24-31, 2015.

[14] S. Sun, B. Rong, and Y. Qian, "Artificial frequency selective channel for covert cyclic delay diversity orthogonal frequency division multiplexing transmission," Security and Communication Networks, vol. 8, no. 9, pp. 1707-1716, 2015.

[15] Q. Li, R. Q. Hu, Y. Qian, and G. Wu, "Cooperative communications for wireless networks: techniques and applications in LTEadvanced systems," IEEE Wireless Communications, vol. 19, no. 2, pp. 22-29, 2012.

[16] M. Boucadair and C. Jacquenet, "Software-defined networking: a perspective from within a service provider environment," RFC 7149, Internet Engineering Task Force, 2014.

[17] X. Chen, R. Q. Hu, and Y. Qian, "Distributed resource and power allocation for device-to-device communications underlaying cellular network," in Proceedings of the IEEE Global Communications Conference (GLOBECOM '14), pp. 4947-4952, Austin, Tex, USA, December 2014.

[18] Z. Zhang, R. Q. Hu, Y. Qian, A. Papathanassiou, and G. Wu, "D2D communication underlay uplink cellular network with fractional frequency reuse," in Proceedings of the 11th International Conference on the Design of Reliable Communication Networks (DRCN '15), pp. 247-250, Kansas City, Mo, USA, March 2015.

[19] L. L. Wei, R. Q. Hu, T. He, and Y. Qian, "Device-to-device(d2d) communications underlaying MU-MIMO cellular networks," in Proceedings of the IEEE Global Communications Conference (GLOBECOM '13), pp. 4902-4907, IEEE, Atlanta, Ga, USA, December 2013.

[20] S. Sun, M. Kadoch, L. Gong, and B. Rong, "Integrating network function virtualization with SDR and SDN for $4 \mathrm{G} / 5 \mathrm{G}$ networks," IEEE Network, vol. 29, no. 3, pp. 54-59, 2015.

[21] B. A. A. Nunes, M. Mendonca, X.-N. Nguyen, K. Obraczka, and T. Turletti, "A survey of software-defined networking: past, present, and future of programmable networks," IEEE Communications Surveys \& Tutorials, vol. 16, no. 3, pp. 16171634, 2014.

[22] R. Guerzoni, I. Vaishnavi, A. Frimpong, and R. Trivisonno, "Virtual link mapping for delay critical services in SDN-enabled 5G networks," in Proceedings of the 1st IEEE Conference on Network Softwarization (NetSoft '15), pp. 1-9, IEEE, London, UK, April 2015.

[23] H.-H. Cho, C.-F. Lai, T. K. Shih, and H.-C. Chao, "Integration of SDR and SDN for 5G," IEEE Access, vol. 2, pp. 1196-1204, 2014.
[24] J. J. Vegas Olmos and I. Tafur Monroy, "Millimeter-wave wireless links for 5G mobile networks," in Proceedings of the 17th International Conference on Transparent Optical Networks (ICTON '15), p. 1, IEEE, Budapest, Hungary, July 2015.

[25] P. Wang, W. Song, D. Niyato, and Y. Xiao, "QoS-aware cell association in $5 \mathrm{G}$ heterogeneous networks with massive MIMO," IEEE Network, vol. 29, no. 6, pp. 76-82, 2015.

[26] Y. Yan, Y. Qian, H. Sharif, and D. Tipper, "A survey on smart grid communication infrastructures: motivations, requirements and challenges," IEEE Communications Surveys and Tutorials, vol. 15, no. 1, pp. 5-20, 2013.

[27] Y. Yan, Y. Qian, H. Sharif, and D. Tipper, "A survey on cyber security for smart grid communications," IEEE Communications Surveys and Tutorials, vol. 14, no. 4, pp. 998-1010, 2012.

[28] K. Lu, Y. Qian, M. Guizani, and H.-H. Chen, "A framework for a distributed key management scheme in heterogeneous wireless sensor networks," IEEE Transactions on Wireless Communications, vol. 7, no. 2, pp. 639-647, 2008.

[29] K. Lu, Y. Qian, and H.-H. Chen, "Wireless broadband access: wimax and beyond-a secure and service-oriented network control framework for wimax networks," IEEE Communications Magazine, vol. 45, no. 5, pp. 124-130, 2007. 

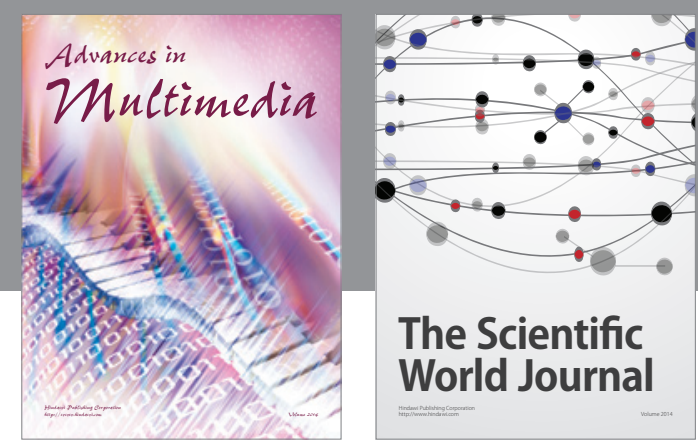

The Scientific World Journal
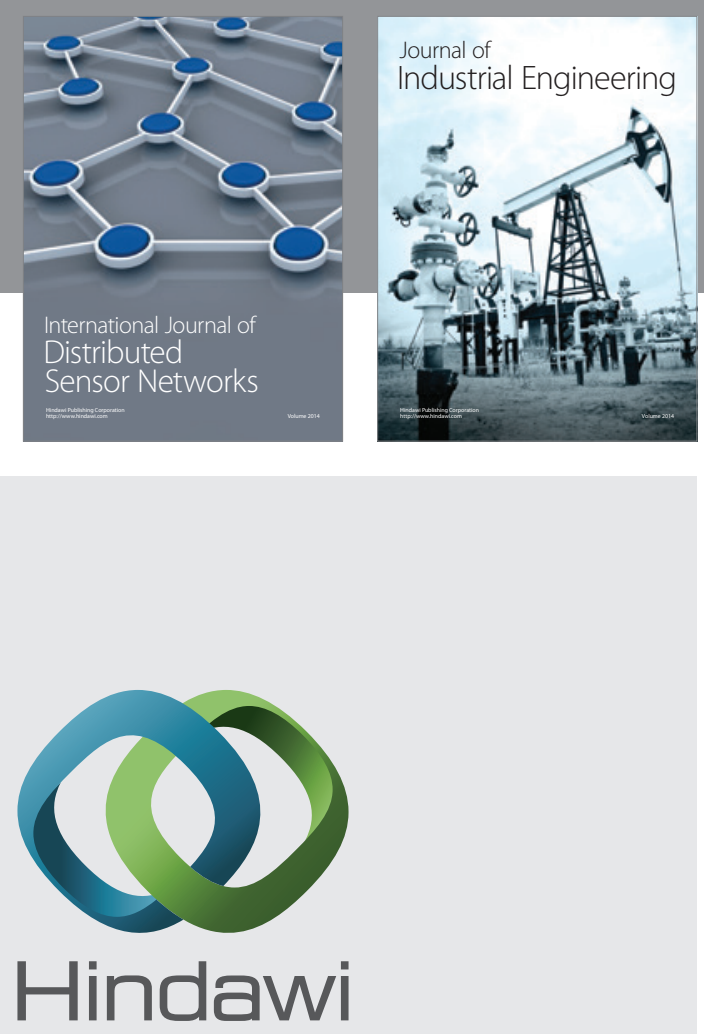

Submit your manuscripts at

http://www.hindawi.com

\section{Computer Networks} and Communications
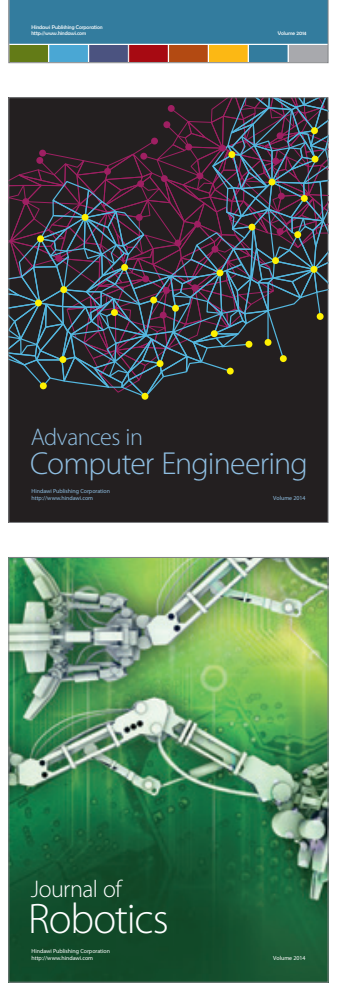
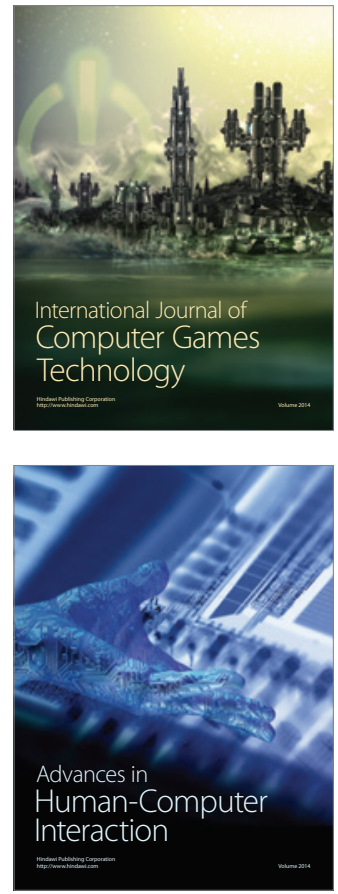
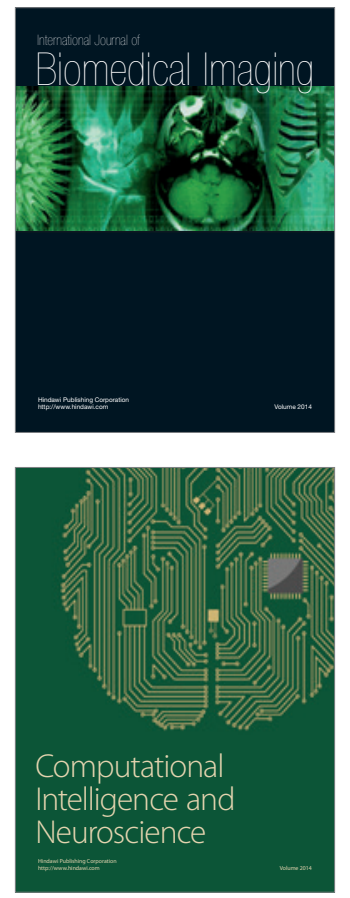
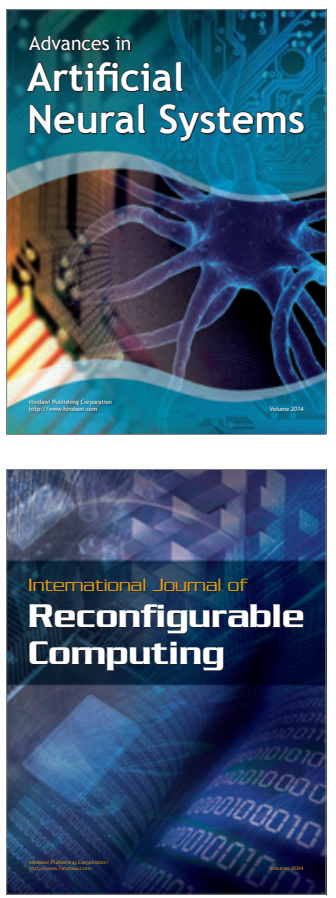
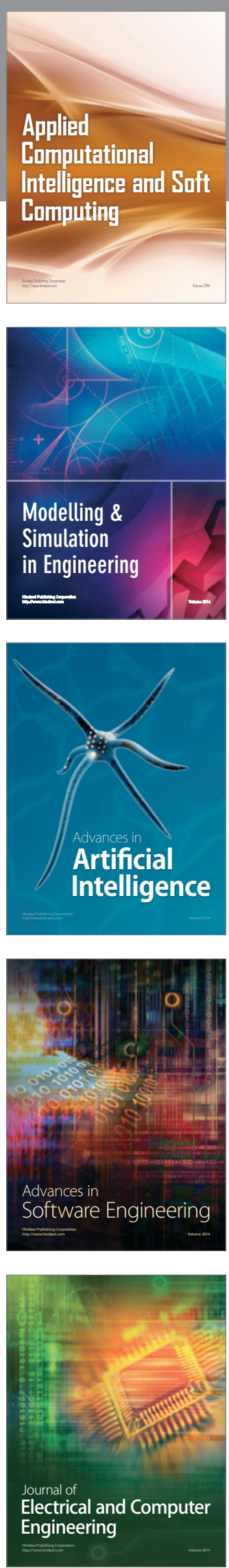